\title{
Giant Faraday rotation in single- and multilayer graphene
}

\author{
Iris Crassee ${ }^{1}$, Julien Levallois ${ }^{1}$, Andrew L. Walter ${ }^{2,3}$, Markus Ostler ${ }^{4}$, Aaron Bostwick ${ }^{3}$, Eli Rotenberg ${ }^{3}$, \\ Thomas Seyller ${ }^{4}$, Dirk van der Marel' ${ }^{1}$ and Alexey B. Kuzmenko ${ }^{1 \star}$
}

The rotation of the polarization of light after passing a medium in a magnetic field, discovered by Faraday ${ }^{1}$, is an optical analogue of the Hall effect, which combines sensitivity to the carrier type with access to a broad energy range. Up to now the thinnest structures showing the Faraday rotation were several-nanometre-thick two-dimensional electron gases ${ }^{2}$. As the rotation angle is proportional to the distance travelled by the light, an intriguing issue is the scale of this effect in two-dimensional atomic crystals or films-the ultimately thin objects in condensed matter physics. Here we demonstrate that a single atomic layer of carbon-graphene-turns the polarization by several degrees in modest magnetic fields. Such a strong rotation is due to the resonances originating from the cyclotron effect in the classical regime and the inter-Landau-level transitions in the quantum regime. Combined with the possibility of ambipolar doping ${ }^{3}$, this opens pathways to use graphene in fast tunable ultrathin infrared magneto-optical devices.

Graphene's unusual physical properties, ${ }^{3,4}$ make it highly attractive for both fundamental research and new applications. Importantly, the conduction and valence bands in graphene show a relativistic massless dispersion and a chiral character of the electronic wavefunctions, resulting in non-equidistant Landau levels (LLs) $(n=0, \pm 1, \pm 2 \ldots)$ :

$$
E_{n}=\operatorname{sign}(n) \sqrt{2 e \hbar v_{\mathrm{F}}^{2}|n B|}
$$

in a perpendicular magnetic field $B$, which includes an anomalous LL at zero energy $E_{0}=0(e>0$ is the elementary charge and $v_{\mathrm{F}} \approx 10^{6} \mathrm{~m} \mathrm{~s}^{-1}$ is the Fermi velocity). As a consequence, graphene shows the half-integer quantum Hall effect ${ }^{5,6}$, first observed in exfoliated graphene flakes ${ }^{7,8}$ and later in graphene epitaxially grown on $\mathrm{SiC}$ (refs 9-11).

The optical properties of graphene are equally unusual. The relativistic and gapless dispersion results in a universal and constant optical conductivity of $\sigma_{0}=e^{2} / 4 \hbar$ (refs $6,12-17$ ) for the photon energies $\hbar \omega$ above twice the Fermi energy $\left(\epsilon_{\mathrm{F}}\right)$. In magnetic field, the non-equidistant spacing of the LLs gives rise to a spectacular series of absorption peaks, corresponding to transitions between various levels ${ }^{18-21}$. In contrast, only one peak, centred at the cyclotron frequency $\omega_{\mathrm{c}}=e B / m_{\mathrm{c}}$, where $m_{\mathrm{c}}$ is the cyclotron mass, is observed in conventional two-dimensional electron gases produced in semiconductor heterostructures.

Up to now, experimental magneto-optical studies of graphene ${ }^{18,19,21}$ have been focused on the diagonal conductivity

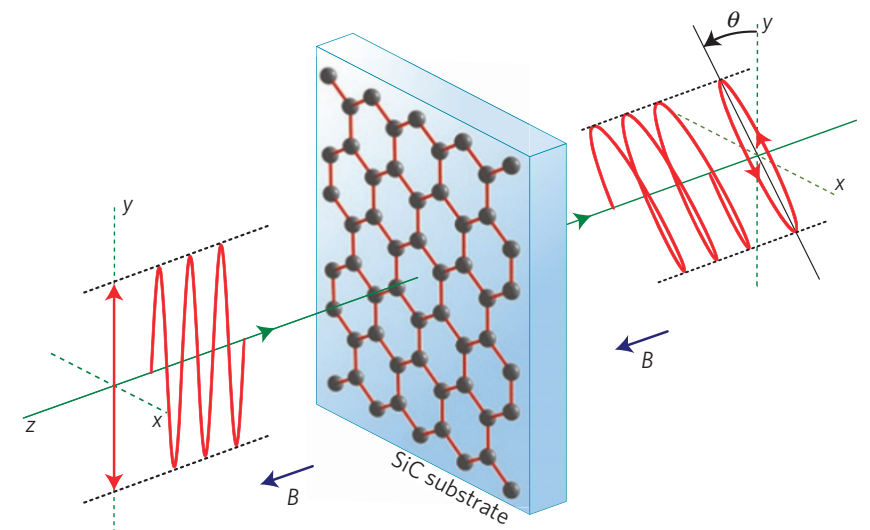

Figure 1 | Schematic diagram of the magneto-optical Faraday-rotation experiment. The polarization plane of the linearly polarized incoming beam is rotated by the Faraday angle $\theta$ after passing through graphene on a $\mathrm{SiC}$ substrate in a perpendicular magnetic field. Simultaneously, the polarization acquires a certain ellipticity. The positive direction of the magnetic field is along the $z$ axis.

component $\sigma_{x x}(\omega, B)$; the off-diagonal, or a.c. Hall ${ }^{22}$, conductivity $\sigma_{x y}(\omega, B)$ was addressed only theoretically ${ }^{20,23,24}$. The diagonal and Hall conductivities are directly related to the absorption $1-T$ and Faraday rotation angle $\theta$ (Fig. 1) respectively. Adopting the thin-film approximation and keeping only the terms linear in conductivity ${ }^{18,23}$, the following expressions are obtained:

$$
\begin{gathered}
1-T(\omega, B) \approx 2 Z_{0} f_{s}(\omega) \operatorname{Re}\left[\sigma_{x x}(\omega, B)\right] \\
\theta(\omega, B) \approx Z_{0} f_{s}(\omega) \operatorname{Re}\left[\sigma_{x y}(\omega, B)\right]
\end{gathered}
$$

where $T$ is the substrate-normalized transmission (see the Methods section), $Z_{0} \approx 377 \Omega$ is the impedance of vacuum and $f_{s}(\omega)$ is a spectrally featureless function specific to the substrate (as described in Supplementary Information). Notably, the diagonal conductivity is formally independent of the sign of the charge carriers, whereas the d.c. Hall conductivity, which is sensitive to the carrier type, lacks spectral information. Therefore, studying the Faraday rotation is needed to complete the picture of the dynamics of electrons and holes in graphene.

Graphene, epitaxially grown on silicon carbide, is well adapted for magneto-optical studies because of its well-controlled

\footnotetext{
${ }^{1}$ Département de Physique de la Matière Condensée, Université de Genève, $\mathrm{CH}-1211$ Genève 4, Switzerland, ${ }^{2}$ Department of Molecular Physics, Fritz-Haber-Institut der Max-Planck-Gesellschaft, Faradayweg 4-6, 14195 Berlin, Germany, ${ }^{3}$ E. O. Lawrence Berkeley Laboratory, Advanced Light Source, MS6-2100, Berkeley, California 94720, USA, ${ }^{4}$ Lehrstuhl für Technische Physik, Universität Erlangen-Nürnberg, Erwin-Rommel-Str. 1, 91058 Erlangen, Germany. *e-mail: Alexey.Kuzmenko@unige.ch.
} 

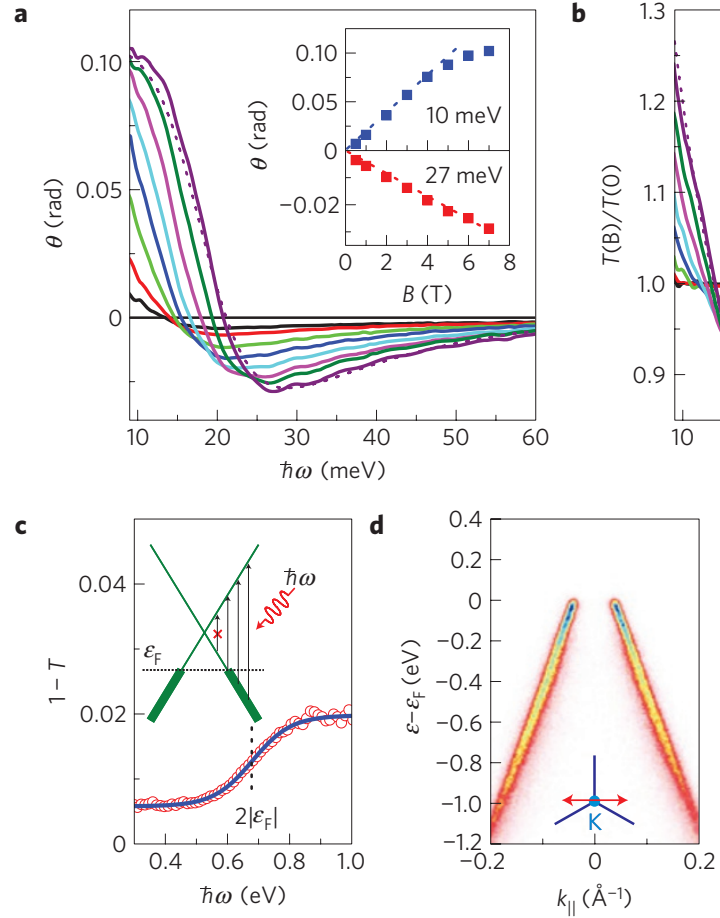

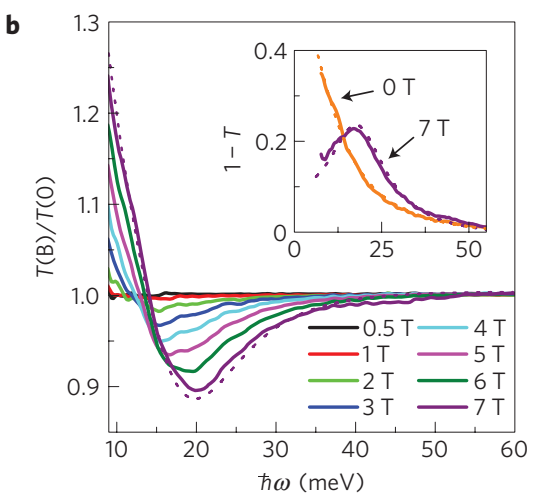

e
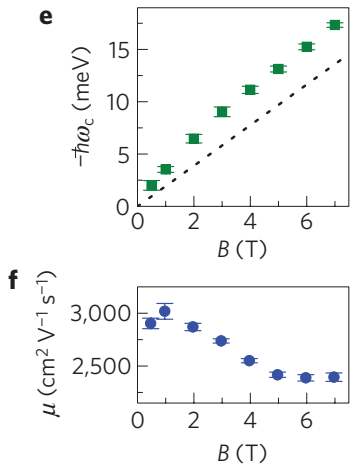

Figure 2 | Faraday rotation and magneto-optical transmission spectra of SLG. $\mathbf{a}$, The Faraday angle $\theta$ at several fields up to $7 \mathrm{~T}$ at $5 \mathrm{~K}$. The inset presents the magnetic-field dependence of $\theta(B)$ at $\hbar \omega=10$ and $27 \mathrm{meV}$. The dashed lines are linear fits of the data points between 0 and $5 \mathrm{~T}$. b, The transmission ratio $T(B) / T(0)$ at the same fields. In the inset the absorption spectra $1-T(B)$ for $B=0 T$ and $7 \mathrm{~T}$ are shown. The dashed lines in both panels and the inset of $\mathbf{b}$ are fits using the classical cyclotron resonance equations (4) and (5). $\mathbf{c}$, The absorption of the same sample at room temperature in zero field (symbols), showing a clear step at $\hbar \omega=2\left|\epsilon_{F}\right|$ due to Pauli blocking as shown in the inset. The blue line is a fitting curve using a phenomenological broadened step function $a+b \tanh \left[\left(\hbar \omega-2\left|\epsilon_{F}\right|\right) / \delta \epsilon\right]$ giving $\left|\epsilon_{F}\right|=0.34 \pm 0.01 \mathrm{eV}$. d, The band dispersion near the K point measured by ARPES on another sample prepared in the same way. $\mathbf{e}$, The cyclotron energy as a function of $B$. The dashed line is a theoretical dependence based on equation (6) using $V_{F}=1.02 \times 10^{6}$ and $\epsilon_{F}=-0.34 \mathrm{eV}$. f , The field dependence of the carrier mobility. The error bars in $\mathbf{e}$ and $\mathbf{f}$ represent the difference between the extreme parameter values obtained from a series of fits of the magneto-optical transmission and Faraday rotation spectra, where the input data were varied arbitrarily within the range of their systematic uncertainties.

morphology and essentially unlimited size ${ }^{25,26}$. Here, we used single- and multilayer graphene (SLG and MLG), grown respectively on the Si-terminated ${ }^{26}$ and C-terminated ${ }^{25}$ surfaces of $6 \mathrm{H}-\mathrm{SiC}$. The first sample underwent hydrogen passivation of the Si dangling bonds ${ }^{27,28}$, resulting in quasifreestanding SLG. The MLG consists of four to six rotated atomic layers (see the Methods section). These samples enabled us to explore the cases of both electron and hole doping as well as to access both the classical (highdoping) and quantum (low-doping) regimes as described below.

Figure $2 \mathrm{a}$ shows the Faraday angle $\theta$ measured on SLG at $5 \mathrm{~K}$ in magnetic fields up to $7 \mathrm{~T}$ in the far-infrared range as described in the Methods section. The spectra show a strongly field-dependent edge-like structure, giving rise to a positive rotation at low energies and negative $\theta$ at high energies. The maximum Faraday rotation exceeds $0.1 \mathrm{rad}\left(\sim 6^{\circ}\right)$, which is an exceptionally large effect given that it comes from a single layer. Measurements on the bare substrate did not reveal any Faraday effect, hence the observed rotation comes exclusively from the carbon monolayer. The inset shows the field dependence of $\theta$ at 10 and $27 \mathrm{meV}$. The curves follow an approximately linear dependence, with opposite slopes of $+18.5 \operatorname{mrad~T}^{-1}$ and $-4.5 \operatorname{mrad~T}^{-1}$ respectively.

The zero-field-normalized transmission spectra $T(B) / T(0)$ of SLG also reveal a strong magnetic-field dependence (Fig. 2b). The inset shows the absorption $(1-T)$ at 0 and $7 \mathrm{~T}$. We can clearly see a strong Drude peak, signalling a high doping level. The peak centre shifts from zero to a finite energy at $7 \mathrm{~T}$, which is due to the cyclotron resonance as we discuss below. Although an estimate of the doping can be made from the integrated intensity of the Drude peak, the most direct measurement of the Fermi energy comes from the absorption spectra in the mid-infrared range (Fig. 2c), where an absorption edge is easily recognized. This is expected to be at $2\left|\epsilon_{\mathrm{F}}\right|$ owing to Pauli blocking ${ }^{6,12,13,16,17}$, giving an estimate for $\left|\epsilon_{\mathrm{F}}\right|=0.34 \pm 0.01 \mathrm{eV}$.

Independently, we have carried out angle-resolved photoemission spectroscopy (ARPES) on a similar sample (Fig. 2d). The obtained dispersion curves show that the sample is p doped (the Fermi level is in the valence band). The Fermi energy inferred from optical measurements is in a reasonable agreement with an extrapolation of the occupied bands measured by ARPES. Below we show that the carrier type can also be directly extracted from the Faraday rotation.

The doping level and field strength used here puts the system in the classical regime, where the separation between the LLs at the Fermi energy is much smaller than $\epsilon_{\mathrm{F}}$ (refs 17,21). In this case, the Dirac quasiparticles are expected to exhibit the classical cyclotron resonance effect. Indeed, the data in Fig. 2a,b at each separate field are well fitted (dashed lines) using equations (2) and (3) and the classical Drude formulae:

$$
\begin{gathered}
\sigma_{x x}(\omega, B)=\frac{2 D}{\pi} \cdot \frac{1 / \tau-i \omega}{\omega_{c}{ }^{2}-(\omega+i / \tau)^{2}} \\
\sigma_{x y}(\omega, B)=-\frac{2 D}{\pi} \cdot \frac{\omega_{c}}{\omega_{c}{ }^{2}-(\omega+i / \tau)^{2}}
\end{gathered}
$$



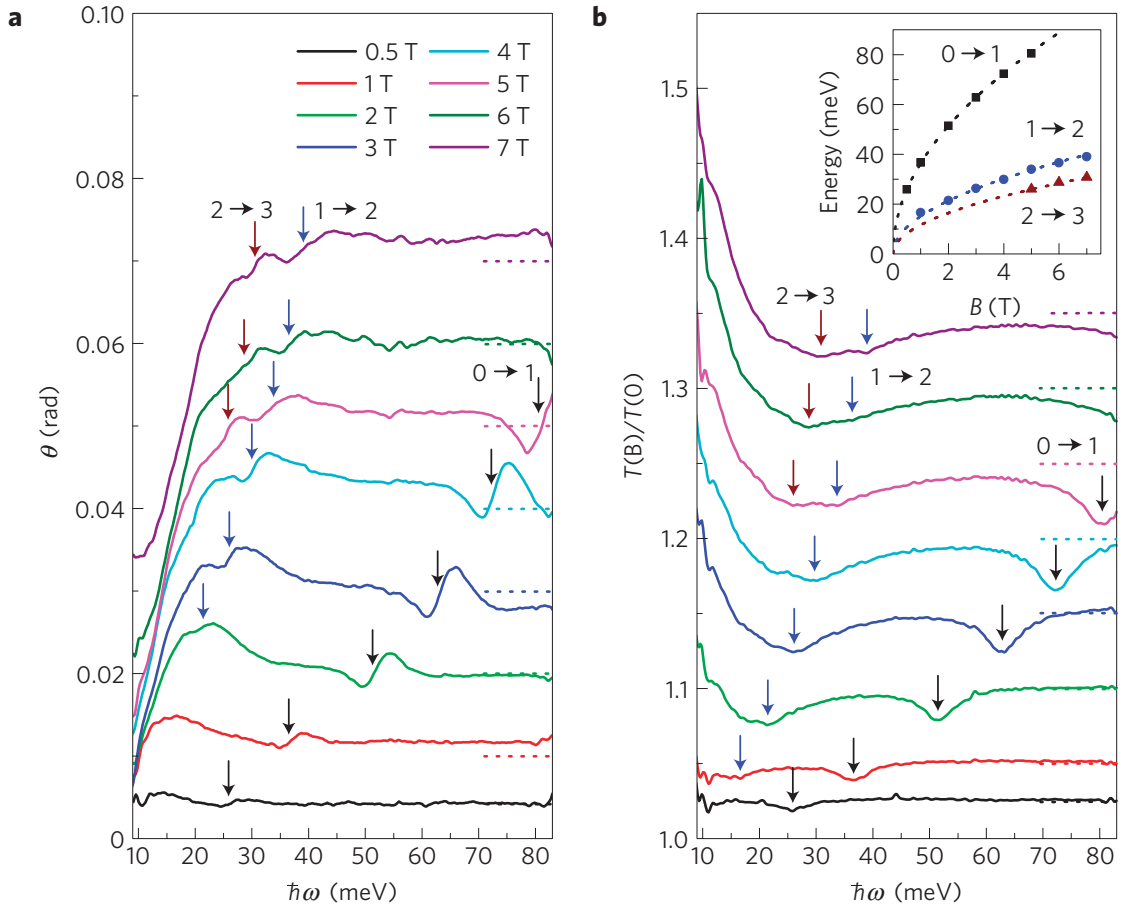

Figure 3 | Faraday rotation and magneto-optical transmission spectra of MLG. a,b, The Faraday angle $\theta(\mathbf{a})$ and the transmission ratio $T(B) / T(0)(\mathbf{b})$ at several fields up to $7 \mathrm{~T}$ at $5 \mathrm{~K}$. The curves in both panels are displaced for clarity; the offsets are given by the dashed lines of the same colours. The energies of the inter-LL transitions $0 \rightarrow 1,1 \rightarrow 2$ and $2 \rightarrow 3$ are indicated by the black, blue and dark red arrows respectively and shown as functions of magnetic field in the inset of $\mathbf{b}$, where theoretical curves obtained using equation (1) with $v_{\mathrm{F}}=1.00 \times 10^{6} \mathrm{~m} \mathrm{~s}^{-1}$ are also shown as dashed lines.

where $D$ is the Drude weight, $\omega_{c}$, the cyclotron frequency, is positive for electrons and negative for holes and $\tau$ is the scattering time. From here we see that the observed large Faraday rotation is associated with the cyclotron resonance. Furthermore, the curve $\theta(\omega)$ reveals the value and sign of the cyclotron frequency, because $\left|\omega_{c}\right|$ coincides with the position of the maximum absolute slope $|\mathrm{d} \theta(\omega) / \mathrm{d} \omega|$, and the sign of the slope matches the sign of $\omega_{c}$. In our case, the negative slope is an unmistakable signature of hole doping.

In the picture of non-interacting Dirac fermions, the Drude weight (at zero magnetic field) is equal to $2 \sigma_{0}\left|\epsilon_{\mathrm{F}}\right| / \hbar$. Using this relation, we estimate $\left|\epsilon_{\mathrm{F}}\right|=0.35 \pm 0.03 \mathrm{eV}$ from the Drude weight extracted from the fit. This agrees with the estimate on the basis of the mid-infrared absorption threshold but is subject to relatively large error bars.

The cyclotron frequency (Fig. 2e) demonstrates an approximately linear growth with field, in agreement with the theoretical relation ${ }^{12,17}$

$$
\omega_{\mathrm{c}}=\frac{e B v_{\mathrm{F}}^{2}}{\epsilon_{\mathrm{F}}}
$$

However, the theoretical curve using the value $\epsilon_{\mathrm{F}}=-0.34 \mathrm{eV}$ and $v_{\mathrm{F}}=1.02 \times 10^{6} \mathrm{~m} \mathrm{~s}^{-1}$ extracted from the mid-infrared optical spectra and ARPES data respectively (dashed line in Fig. 2e) is somewhat lower than the experiment. Although the reason for this deviation is currently unknown, we can speculate about its possible relation to many-body effects. Interestingly, an electron-plasmon coupling was recently observed in similar samples ${ }^{29}$.

The carrier mobility $\mu$ can also be extracted using the relation $\left|\omega_{\mathrm{c}}\right| \tau=\mu|B|$ (Fig. 2f). The mobility varies weakly with field between 2,400 and $3,000 \mathrm{~cm}^{2} \mathrm{~V}^{-1} \mathrm{~s}^{-1}$. Even at $7 \mathrm{~T}, \mu|B| \sim 1$, which means that the cyclotron resonance is significantly damped owing to disorder. This can also be seen from the large width $(1 / \tau \sim 10 \mathrm{meV})$ of the cyclotron peak in the inset of Fig. $2 \mathrm{~b}$.
The Faraday rotation and absorption observed in MLG are strikingly different (Fig. 3). Both the Faraday angle and absorption spectra show extra, strongly field-dependent, resonance structures, marked by arrows. As was found in previous studies ${ }^{18,19}$, they correspond to optical transitions between individual LLs. The series of transitions with energies $E_{1}-E_{0}, E_{2}-E_{1}$ and $E_{3}-$ $E_{2}$ can be identified and fitted using equation (1), which gives $v_{\mathrm{F}}=1.00 \pm 0.01 \times 10^{6} \mathrm{~m} \mathrm{~s}^{-1}$ (inset of Fig. 3b). Although inter-LL absorption peaks have been observed before ${ }^{18,19}$, the measurement of the Faraday rotation in the same conditions is the key novelty of the present work. Now we can distinguish transitions involving electrons from ones involving holes. In particular, the positive sign of $\mathrm{d} \theta(\omega) / \mathrm{d} \omega$ at the transition energies, which coincide with the inflection points, unequivocally determines that the transitions are between electron bands. The low-frequency part of the spectra features a cyclotron-resonance-like structure, similar to the data on SLG. The sign of the Faraday angle now corresponds to electron doping.

As only transitions between occupied and empty levels are allowed, the simultaneous presence of $0 \rightarrow 1,1 \rightarrow 2$ and $2 \rightarrow 3$ transitions and the low-frequency cyclotron structure in MLG arises from a variation of the Fermi energy across different layers. The layer closest to the substrate is highly doped ${ }^{30}$; the doping in subsequent layers decreases exponentially with layer number. The most strongly doped innermost layer gives rise to the cyclotron resonance, as in SLG. Meanwhile, the individual inter-LL transitions originate from the weakly doped layers, which are in the quantum regime, as can be seen from the square-root field dependence of the transition energies. The maximum Faraday angle in this sample is smaller than in the monolayer sample, because the innermost layer in MLG is more weakly doped.

Summarizing the data on both samples, we point out that the Faraday rotation is a powerful contact-free tool to distinguish the carrier type and measure the mobility in graphene. In both the classical (high-doping) and quantum (low-doping) 
limits this technique reveals magneto-optical resonances owing to either the cyclotron effect or to inter-LL transitions. The technique has the potential to distinguish electronic contributions from separate graphene layers. However, our most striking observation is the giant value of the rotation $(>0.1 \mathrm{rad}$ for a single atomic layer). This is much larger than the fine-structure constant $\left(\sim 10^{-2}\right)$ - the predicted scale for the Faraday angle, associated with the quantized Hall conductance ${ }^{23,24,31,32}$. We have demonstrated that this enhancement is due to the proximity to the resonance frequency. Although in two-dimensional electron gases the cyclotron resonance gives rise to comparable absolute rotations ${ }^{2,32}$, the rotation comes from an effective layer that is about one order of magnitude thicker than graphene. Furthermore, although in two-dimensional electron gases the cyclotron frequency is doping independent, in graphene it can be tuned with doping (electrostatic or chemical) as seen from equation (6), and much higher frequencies can be achieved at the same fields.

The charge scattering determines the width of the cyclotron peak and inter-LL transitions. Improving the mobility will increase the rotations observed. As in the classical limit the Fermi-Dirac thermal filling factors play a minor role in the optical conductivity, the amount of rotation in this regime is not expected to be significantly reduced at elevated temperatures.

The Faraday effect and the associated magneto-optical Kerr effect are widely used in optical communication, data storage and computing. We suggest that the use of graphene for fast tunable ultrathin magneto-optical devices should be explored. Indeed, the possibility of easy and fast ambipolar doping together with a strong Faraday effect in a wide frequency range is a unique combination not present in other known materials.

\section{Methods}

Samples. Single- and multilayer epitaxial graphene were made by graphitization of the surface of semi-insulating $6 \mathrm{H}-\mathrm{SiC}$ substrates in an argon atmosphere ${ }^{26}$. The sample size was $10 \times 10 \mathrm{~mm}^{2}$. SLG was obtained on the Si-terminated face of SiC by first preparing a $(6 \sqrt{3} \times 6 \sqrt{3})$ reconstructed surface (the so-called buffer layer) by annealing in 1 bar of Ar at a temperature of $1,450^{\circ} \mathrm{C}$, which was subsequently converted into quasifreestanding graphene by intercalation of hydrogen ${ }^{27,28}$. MLG was grown on the C-terminated face of SiC. In either case, the back side of the substrate was cleaned from undesirably grown graphene using Scotch tape and was checked to be graphene free using X-ray photo-emission. Likewise, the layer thicknesses of the SLG and MLG samples were determined using X-ray photo-emission measurements.

Magneto-optical experiment. The sample was mounted in a split-coil superconducting magnet attached to a Fourier-transform spectrometer. A Globar light source and He-cooled bolometer detector were used. Two grid-wire gold polarizers (one fixed and one rotating) were mounted before and after the sample. The Faraday rotation was deduced from the angle of the rotating polarizer at which the intensity was at minimum. The substrate-normalized transmission $T$ was measured with one polarizer only and is defined as the ratio between the intensity of the light transmitted through the graphene on $\mathrm{SiC}$ and through bare substrate.

Received 17 May 2010; accepted 14 September 2010; published online 7 November 2010

\section{References}

1. Faraday, M. On the magnetization of light and the illumination of magnetic lines of force. Phil. Trans. R. Soc. 136, 104-123 (1846).

2. Suzuki, M., Fujii, K., Ohyama, T., Kobori, H. \& Kotera, N. Far-infrared resonant Faraday effect in semiconductors. J. Phys. Soc. Jpn 72, 3276-3285 (2003).

3. Geim, A. K. \& Novoselov, K. S. The rise of graphene. Nature Mater. 6, 183-191 (2007).

4. Castro Neto, A. H., Guinea, F., Peres, N. M. R., Novoselov, K. S. \& Geim, A. K. The electronic properties of graphene. Rev. Mod. Phys. 81, 109-162 (2009).

5. Gusynin, V. P. \& Sharapov, S. G. Unconventional integer quantum Hall effect in graphene. Phys. Rev. Lett. 95, 146801 (2005).

6. Peres, N. M. R., Guinea, F. \& Castro Neto, A. H. Electronic properties of disordered two-dimensional carbon. Phys. Rev. B 73, 125411 (2006).

7. Novoselov, K. S. et al. Two-dimensional gas of massless Dirac fermions in graphene. Nature 438, 197-200 (2005).
8. Zhang, Y., Tan, Y-W., Stormer, H. L. \& Kim, P. Experimental observation of the quantum Hall effect and Berry's phase in graphene. Nature 438, 201-204 (2005).

9. Jobst, J. et al. Quantum oscillations and quantum Hall effect in epitaxial graphene. Phys. Rev. B 81, 195434 (2010).

10. Shen, T. et al. Observation of quantum-Hall effect in gated epitaxial graphene grown on SiC (0001). Appl. Phys. Lett. 95, 172105 (2009).

11. Wu, X. et al. Half integer quantum Hall effect in high mobility single layer epitaxial graphene. Appl. Phys. Lett. 95, 223108 (2009).

12. Ando, T., Zheng, Y. \& Suzuura, H. Dynamical conductivity and zero-mode anomaly in honeycomb lattices. J. Phys. Soc. Jpn 71, 1318-1324 (2002).

13. Falkovsky, L. A. \& Varlamov, A. A. Space-time dispersion of graphene conductivity. Eur. Phys. J. B 56, 281-284 (2007).

14. Kuzmenko, A. B., van Heumen, E., Carbone, F. \& van der Marel, D. Universal optical conductance of graphite. Phys. Rev. Lett. 100, 117401 (2008).

15. Nair, R. R. et al. Fine structure constant defines visual transparancy of graphene. Science 320, 1308 (2008)

16. Li, Z. Q. et al. Dirac charge dynamics in graphene by infrared spectroscopy. Nature Phys. 4, 532-535 (2008).

17. Gusynin, V. P., Sharapov, S. G. \& Carbotte, J. P. On the universal AC optical background in graphene. New J. Phys. 11, 095013 (2009).

18. Sadowski, M. L., Martinez, G., Potemski, M., Berger, C. \& de Heer, W. A. Landau level spectroscopy of ultrathin graphite layers. Phys. Rev. Lett. 97, 266405 (2006)

19. Jiang, Z. et al. Infrared spectroscopy of Landau levels in graphene. Phys. Rev. Lett. 98, 197403 (2007)

20. Gusynin, V. P., Sharapov, S. G. \& Carbotte, J. P. Magneto-optical conductivity in graphene. J. Phys. Condens. Matter 19, 026222 (2007).

21. Orlita, M. et al. Approaching the Dirac point in high-mobility multilayer epitaxial graphene. Phys. Rev. Lett. 101, 267601 (2008).

22. Kaplan, S. G. et al. Normal state ac Hall effects in $\mathrm{YBa}_{2} \mathrm{Cu}_{3} \mathrm{O}_{7}$ thin films. Phys. Rev. Lett. 76, 696-699 (1996).

23. Morimoto, T., Hatsugai, Y. \& Aoki, H. Optical Hall conductivity in ordinary and graphene quantum Hall systems. Phys. Rev. Lett. 103, 116803 (2009).

24. Fialkovsky, I. V. \& Vassilevich, D. V. Parity-odd effects and polarization rotation in graphene. J. Phys. A 42, 442001 (2009).

25. Berger, C. et al. Ultrathin epitaxial graphite: 2 d electron gas properties and a route toward graphene-based nanoelectronics. J. Phys. Chem. B 108, 19912-19916 (2004).

26. Emtsev, K. V. et al. Towards wafer-size graphene layers by atmospheric pressure graphitization of silicon carbide. Nature Mater. 8, 203-207 (2009).

27. Riedl, C., Coletti, C., Iwasaki, T., Zakharov, A. A. \& Starke, U. Quasi-free-standing epitaxial graphene on SiC obtained by hydrogen intercalation. Phys. Rev. Lett. 103, 246804 (2009).

28. Speck, F. et al. Quasi-freestanding graphene on $\mathrm{SiC}(0001)$. Mat. Sci. Forum 645-648, 629-632 (2010)

29. Bostwick, A. et al. Observation of plasmarons in quasi-free-standing doped graphene. Science 328, 999-1002 (2010).

30. Ohta, T. et al. Interlayer interaction and electronic screening in multilayer graphene investigated with angle-resolved photoemission spectroscopy. Phys. Rev. Lett. 98, 206802 (2007).

31. Volkov, V. A. \& Mikhailov, S. A. Quantization of the Faraday-effect in systems with a quantum Hall-effect. JETP Lett. 41, 476-478 (1985).

32. Ikebe, Y. et al. Optical Hall effect in the integer quantum Hall regime. Phys. Rev. Lett. 104, 256802 (2010).

\section{Acknowledgements}

This work was supported by the Swiss National Science Foundation (SNSF) by the grants 200021-120347 and IZ73Z0-128026 (SCOPES program), through the National Centre of Competence in Research 'Materials with Novel Electronic Properties-MaNEP'. Work in Erlangen was supported by the German Research Council (DFG) through research grant SE 1087/5-1 and through the Cluster of Excellence 'Engineering of Advanced Materials' at the University of Erlangen-Nuremberg. We thank S. G. Sharapov, J. Hancock and J. L. M. van Mechelen for discussions.

\section{Author contributions}

I.C. made magneto-optical measurements, analysed data and wrote the manuscript. J.L. made magneto-optical measurements and discussed data; M.O. and A.L.W. carried out the growth of the $6 \sqrt{3}$ reconstruction on the Si face and the multilayer on the $\mathrm{C}$ face. T.S. carried out the hydrogen intercalation, the removal of graphene from the back side of the samples and the XPS characterization. A.B. and E.R. made ARPES measurements; D.v.d.M. discussed data; A.B.K. proposed the experiment, made magneto-optical measurements, analysed data and wrote the manuscript.

\section{Additional information}

The authors declare no competing financial interests. Supplementary information accompanies this paper on www.nature.com/naturephysics. Reprints and permissions information is available online at http://npg.nature.com/reprintsandpermissions. Correspondence and requests for materials should be addressed to A.B.K. 